\title{
The Impact of Using Multiple Antennas on Wireless Localization
}

\author{
Konstantinos Kleisouris*, Yingying Chen ${ }^{\dagger}$, Jie Yang ${ }^{\dagger}$, Richard P. Martin* \\ ${ }^{*}$ Dept. of CS and WINLAB, Rutgers University \\ ${ }^{\dagger}$ Dept. of ECE, Stevens Institute of Technology \\ 110 Frelinghuysen Rd, Piscataway, NJ 08854 \\ \{kkonst,rmartin\}@cs.rutgers.edu \\ Castle Point on Hudson, Hoboken, NJ 07030 \\ \{yingying.chen, jyang\}@stevens.edu
}

\begin{abstract}
We show that signal strength variability can be reduced by employing multiple low-cost antennas at fixed locations. We further explore the impact of this reduction on wireless localization by analyzing a representative set of algorithms ranging from fingerprint matching, to statistical maximum likelihood estimation, and to multilateration. We provide experimental evaluation using an indoor wireless testbed of the localization performance under multiple antennas. We found that in nearly all cases the performance of localization algorithms improved when using multiple antennas. Specifically, the median and the 90th percentile error can be reduced up to $70 \%$. Additionally, we found that multiple antennas improve the localization stability significantly, up to $100 \%$ improvement, when there are small scale 3-dimensional movements of a mobile device around a given location.
\end{abstract}

\section{INTRODUCTION}

Accurate and cost effective localization of wireless devices would enable a variety of diverse new applications in areas including health care, inventory control, personal management, robotic navigation and geometric routing. However, indoor environments are particularly challenging for radio-based localization, because effects such as reflection, refraction, shadowing, scattering, make signal characterization with respect to location difficult. The positioning of wireless devices indoors thus remains an active area of research.

To date, most wireless localization systems, based on commercially available components, use received signal strength (RSS) as the base modality. However, a significant problem with RSS is that small-scale multipath fading adds high frequency components with large amplitudes to the signal at a given location. Thus, the RSS can vary by 5-10 dB with small (a few wavelengths) changes in location. We confirm these results in this work. However, because the small-scale fading effects occur at the level of several wavelengths (about $12 \mathrm{~cm}$ at $2.4 \mathrm{GHz}$ ), and the granularity of the localization system is typically much larger (2-3 meters), using multiple receivers spaced on the order of a few wavelengths presents the opportunity to smooth out these effects, while maintaining the same number of landmarks used by the localization system. In particular, multiple receivers can be realized by multiplexing between multiple antennas on a single receiver.

This work investigates the impact on the localization system of using multiple receivers spaced closely together. We performed a trace-driven study on an 802.11 testbed in a real office building environment. Each receiver location, called a landmark, supported 3 antennas spaced within 1-2 ft of each other. We first investigated signal variability, and found that using multiple antennas resulted in signal-to-distance models with better fits to a theoretical curve based on free-space models than when using a single antenna, thus confirming that additional antennas help average out small-scale environmental effects.

We then evaluated the effects of using multiple antennas on wireless localization. In order to evaluate the generality of applying multiple antennas, we evaluated the impact of multiple antennas on a diverse set of algorithms, which use an array of techniques ranging from nearest neighbor matching in signal space, represented by RADAR [1], to statistical maximum likelihood estimation, as represented by the Area-Based Probability (ABP) [2], and to multilateration, represented by Bayesian Networks (BNs) [3]. We found that all algorithms under study improved their absolute position accuracy when using multiple antennas. Another key finding is that using multiple antennas significantly reduces the fraction of poor localization results across almost all of the algorithms. In one case, the median and the 90th percentile localization error were reduced up to $70 \%$.

In addition to accuracy, we also investigated stability. We define stability as the localization system's ability to maintain a position in the face of small-scale movements of a device. For example, if a device moves $1 \mathrm{ft}$, ideally the localization system should return a result that is $1 \mathrm{ft}$ away from the previous position. Instability is a common anecdotal problem with localization systems, but has not received much attention by the research community. We thus conducted a detailed evaluation of the impact of multiple antennas on the localization stability. We quantified how much the localized position of a device moves in the physical space as a function of smallscale movements of the device around its current position. Our results show that multiple antennas help improve localization stability significantly. Specifically, we can achieve up to $100 \%$ improvement in stability over the single antenna case.

A third set of experiments examined how averaging or not averaging the data from multiple antennas at a landmark position impacted the results. If averaging has no measurable impact, then a host using multiple antennas could save bandwidth and computational resources by averaging the RSS values at a single landmark location before localization occurred. However, we found that there is not clear trend whether or not to average the data from multiple antennas.

The final set of experiments explored the algorithms' sen- 
sitivity to the assumption that RSS follows a Gaussian distribution. The main reason to make such an assumption is that it makes the mathematics tractable, because the Gaussian distribution is closed under summation, i.e., the sum of two Gaussians is a Gaussian. This property also allows for averaging of multiple antenna streams to rest on a sound theoretical foundation. We generated synthetic traces that followed a Gaussian distribution using parameters from fitted measured data. Our results show that the performance behavior on real data is consistent with the localization performance under Gaussian distribution for RSS at each testing position.

The rest of the paper is organized as follows. Section II discusses previous research in localization and related antenna work. We present our testbed infrastructure, accuracy and stability metrics as well as our methodology for a series of investigations in Section III. In Section IV we present our experimental results. Specifically, we show the goodness of fit of RSS data to a theoretic model under multiple antennas, and we describe the accuracy and stability performance of localization using RADAR, ABP and BNs with real and Gaussian fingerprint sets. We provide a discussion in Section V. Finally, we conclude our work in Section VI.

\section{RELATED WORK}

There have been active research efforts in positioning wireless devices indoors. Among these, improving localization accuracy is the center focus, ranging from algorithm development, to landmark placement, and to increasing the landmark density. Various localization schemes [1], [2], [3], [4], [5] utilizing different modalities, such as RSS and Time-DifferentOf-Arrival (TDOA), and different mapping functions, such as fingerprint matching and statistical approaches, have been developed to more accurately position mobile devices. [6] investigates the impact of landmark placement on localization performance and proposes an optimal landmark deployment approach to improve the performance without increasing the number of landmarks (about 1 landmark per 4000 square feet). On the other hand, [7] shows that by using the truncated singular value decomposition technique and increasing the landmark density (about 1 landmark per 1000 square feet) a better localization accuracy is achieved. In this work, we take a different approach by exploring the impact on the localization system when using multiple antennas at a given location.

Work that is closely related to ours is [8] and [9]. [8] presents a detailed characterization of signal strength behavior in an 802.15.4 network environment with monopole antennas. Their findings demonstrate that the relative antenna orientation between receiver-transmitter pairs is a major factor in signal strength variability, even in the absence of multipath effects. Further, [9] reviews the principles of radio propagation in indoor environments and also explores relevant concepts such as spatial and temporal variations of the channel, large scale path losses and mean excess delay. Theoretical distributions of the sequences of arrival times, amplitudes and phases are presented. Our work is different in that in addition to a signal variability study, we investigate the impact of using

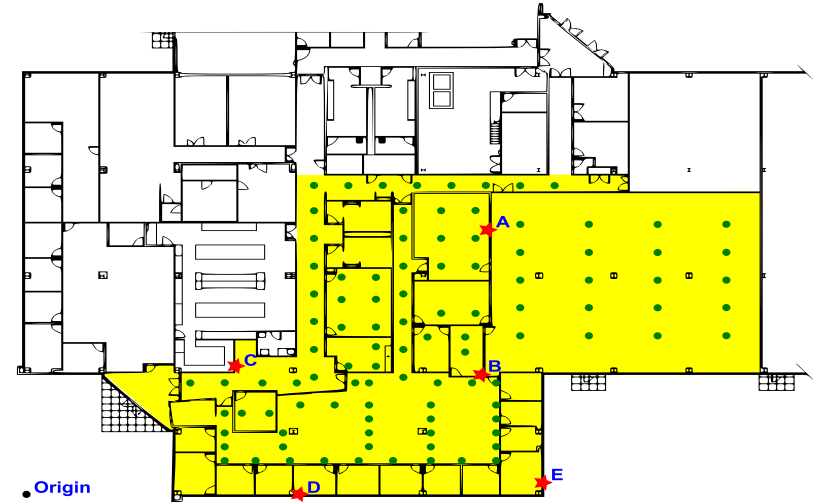

Fig. 1. WINLAB floor plan.

\begin{tabular}{|c|c|c|c|c|c|}
\hline Location & Landmark & Antenna & $\mathrm{x}$ & $\mathbf{y}$ & $\mathbf{z}$ \\
\hline \multirow{3}{*}{$A$} & 1 & 1 & 136 & 96 & 6.25 \\
\hline & \multirow[t]{2}{*}{2} & $\overline{2}$ & 134 & 96 & 6.25 \\
\hline & & 12 & 135 & 96 & 6.25 \\
\hline \multirow{3}{*}{$B$} & 3 & 3 & 131 & 43 & 5 \\
\hline & \multirow{2}{*}{4} & 4 & 134 & 43 & 5 \\
\hline & & 14 & 134 & 43 & 6 \\
\hline \multirow{3}{*}{$C$} & 5 & 5 & 62 & 48.5 & 7.41 \\
\hline & \multirow{2}{*}{6} & 6 & 62 & 46.5 & 7.41 \\
\hline & & 16 & 62 & 47.5 & 7.41 \\
\hline \multirow{3}{*}{$D$} & 7 & 7 & 83 & 1 & 5.83 \\
\hline & \multirow{2}{*}{8} & 8 & 81 & 1 & 5.83 \\
\hline & & 18 & 82 & 1 & 5.83 \\
\hline \multirow{3}{*}{$E$} & 9 & 9 & 151 & 5 & 5.83 \\
\hline & \multirow{2}{*}{10} & $\overline{10}$ & 149 & 1 & 5.83 \\
\hline & & 20 & 148 & 1 & 5.83 \\
\hline
\end{tabular}

TABLE I

Coordinates $x, y, z$ (in feet) of the 15 antennas in our testbed. Locations $A$, $B, C, D, E$ are depicted as red stars in Figure 1.

multiple antennas on wireless localization including accuracy and stability.

Finally, there has been a wide range of research covering development of antennas suitable for mobile communications systems, and many experimental results have been reported to show the system requirements and feasibility. An application of phased-array and adaptive antennas has been suggested in recent years for mobile communications to overcome the problems of single-antenna systems [10], [11], [12], [13], [14], [15], [16], [17]. Specifically, these two types of antennas have been shown to help improve a mobile system's performance in several ways, such as by increasing channel capacity and spectrum efficiency, extending range coverage, reducing cochannel interference and multipath fading.

\section{Methodology}

In this section we describe our experimental methodology. We first describe the infrastructure we used, and then describe the metrics to quantify the localization accuracy and stability. We then present our methodology for a series of investigations, which include: 1) the impact of small-scale movements on localization accuracy and stability, 2) the impact of averaging or not averaging RSS data on a single landmark, 3) the effects of modeling RSS as a Gaussian distribution at a testing location. 


\begin{tabular}{|l|l|l|}
\hline \multicolumn{2}{|l|}{ Placement } & Coordinates, Description \\
\hline \hline Floor & Center & $(x, y, 0)$ \\
\hline \multirow{4}{*}{ Desk } & East & $(x, y, 3)$ \\
\cline { 2 - 3 } & West & $(x+1, y, 3)$ \\
\cline { 2 - 3 } & North & $(x, y+1,3)$ \\
\cline { 2 - 3 } & South & $(x, y-1,3)$ \\
\cline { 2 - 3 } & Vertical & $\begin{array}{l}(x, y, 3), \text { keyboard and monitor vertical to the } \\
\text { floor with Orinoco card pointing to the ceiling }\end{array}$ \\
\cline { 2 - 3 } & Parallel & $\begin{array}{l}(x, y, 3), \text { keyboard vertical to the floor, moni- } \\
\text { tor parallel to the floor }\end{array}$ \\
\hline Shoulder & $(x, y, 5.16)$ \\
\hline
\end{tabular}

TABLE II

Placements of a mobile around a given location $(x, y, z)$ (coordinates in feet). Each location $(x, y, z)$ is depicted as a green dot in Figure 1.

\section{A. Testbed Infrastructure}

All data was collected using an 802.11 (Wi-Fi) network in the Wireless Network Laboratory (WINLAB) at Rutgers University. Figure 1 depicts the floor plan of our experimental site, where the floor size is $219 \mathrm{ft} \times 169 \mathrm{ft}$. All experiments were conducted in the yellow/shaded area, which is the WINLAB space. There are 10 landmarks (also called access points, anchors, or base-stations) deployed at five different locations with 2 landmarks per location. In our terminology, a landmark is any device that observes packet traffic properties, e.g., RSS, at a known location.

The location of each landmark is shown as a red star in Figure 1 and denoted as $A, B, C, D$, and $E$. Each landmark is a Linux machine with a $1-\mathrm{GHz}$ CPU, $512 \mathrm{MBs}$ of RAM and a 20-GB disk. At each location, one landmark has two Atheros miniPCI 802.11 wireless cards, whereas the other only one of the same type. Each card can be connected to an external $7 \mathrm{dBi}$ Omni directional antenna. Thus, there can be up to 3 antennas per location or 15 antennas total. Table I presents the $x, y$ and $z$ coordinates of all antennas along with their numerical IDs.

The green dots in Figure 1 are a total of 101 testing spots where we collected RSS data for testing. For each testing spot $(x, y, z)$, we collected measurements from 7 unique positions with an additional 2 orientations, for a total of 9 unique placements. Table II summarizes all the different placements of a mobile device around a given testing spot. The mobile we consider here is a Dell laptop running Linux and equipped with an Orinoco silver card. Along the height dimension, we call the placements at $0 \mathrm{ft}, 3 \mathrm{ft}$ and $5.16 \mathrm{ft}$ as floor, desk and shoulder respectively. At the desk level, we call the small 1foot movements around the main center placement the north, south, east and west placements. Finally, we call the two orientations the vertical and parallel placements.

For each placement $i$, we estimated an RSS vector $\overline{S_{i}}=$ $\left(\overline{s_{i 1}}, \overline{s_{i 2}}, \ldots, \overline{s_{i j}}, \ldots\right)$. This vector is called a fingerprint, where $\overline{s_{i j}}$ is the average RSS corresponding to antenna $j$ (value of $j$ is based on Table I). Given the number of testing spots and placements around each spot, the total number of fingerprints in our experimental data set is $101 \times 9=909$.

To compute a fingerprint, our mobile would transmit packets and every landmark would forward the packets observed from all the antennas to a centralized server. It would wait for

\begin{tabular}{|c|c|}
\hline Combination & Description \\
\hline$\overline{~ 1-\text { antenna }}$ & $\begin{array}{l}\text { Use the RSS of the landmarks with only one } \\
\text { antenna (i.e., } 1,3,5,7,9 \text { ) }\end{array}$ \\
\hline 2-antenna-noavg & $\begin{array}{l}\text { - Use the RSS of the landmarks with only one } \\
\text { antenna } \\
\text { - Use the RSS of the antenna with smaller ID } \\
\text { from the landmarks with two antennas } \\
\text { (i.e., } 1,2,3,4,5,6,7,8,9,10 \text { ) }\end{array}$ \\
\hline 2-antenna-avg & $\begin{array}{l}\text { Average the RSS of the landmarks with two } \\
\text { antennas (i.e., } \operatorname{avg}(2,12), \operatorname{avg}(4,14), \operatorname{avg}(6 \text {, } \\
16), \operatorname{avg}(8,18), \operatorname{avg}(10,20))\end{array}$ \\
\hline 2-antenna-avg-plus-1 & $\begin{array}{l}\text { - Use the RSS of the landmarks with only one } \\
\text { antenna } \\
\text { - Average the RSS from the landmarks with } \\
\text { two antennas } \\
\text { (i.e., } 1, \operatorname{avg}(2,12), 3, \operatorname{avg}(4,14), 5, \operatorname{avg}(6,16) \text {, } \\
7, \operatorname{avg}(8,18), 9, \operatorname{avg}(10,20))\end{array}$ \\
\hline 3-antenna-noavg & $\begin{array}{l}\text { Use the RSS from the three antennas that exist } \\
\text { at each landmark position } \\
\text { (i.e., } 1,2,3,4,5,6,7,8,9,10,12,14,16,18 \text {, } \\
\text { 20) }\end{array}$ \\
\hline 3-antenna-avg & $\begin{array}{l}\text { Average the RSS from the three antennas that } \\
\text { exist at each landmark position } \\
\text { (i.e., avg }(1,2,12), \operatorname{avg}(3,4,14), \operatorname{avg}(5,6,16) \text {, } \\
\text { avg }(7,8,18), \operatorname{avg}(9,10,20))\end{array}$ \\
\hline
\end{tabular}

TABLE III

Localization antenna combinations for a given landmark position.

at least 350 packets from each antenna before computing a fingerprint. We used the GRAIL infrastructure [18] to collect the packets.

We examined the effect of the orientation of multiple antennas and found it had little overall impact on the RSS differences between multiple antennas at one location. Specifically, we collected fingerprints with all 3 antennas at each landmark location vertical to the floor (the top of the antennas pointing to the ceiling), and another set with one of the 3 antennas being parallel to the floor. After analyzing the two data sets, we concluded that there is no significant difference on the RSS in the two data sets, and thus the results we present in this work are based on data collected with all landmark antennas being vertical to the floor.

\section{B. Metrics}

In this section we formalize our two metrics that apply to all localization algorithms:

Accuracy: For a given localization attempt, accuracy is the Euclidean distance between the location estimate obtained from the localization system and the actual location of the mobile device in the physical space. We refer to this distance as localization error. To capture the statistical characterization of the localization error, we study the Cumulative Distribution Function (CDF) of the localization error for all the testing placements.

Stability: Stability measures how much the location estimate moves in the physical space in response to small-scale movements of a mobile device. We believe that stability is a desirable property in localization systems, since a location estimate should not move too far in the physical space if there is a small-scale movement of a mobile device. For instance, when someone works at his office desk and moves his laptop $1 \mathrm{ft}$ away, the localized position of the laptop should not 
change too much. Thus, we would like to know how stability is affected by using multiple antennas at each landmark.

We define stability by taking the Euclidean distance between the location estimate, $p_{1}$, of a mobile device at its "original" position and the localization results $p_{2}, p_{3}, \ldots, p_{n}$ obtained when the mobile device is moved around its original location. In essence, if $p_{1}$ and $p_{i}(i \neq 1)$ are $k$ feet apart, stability tells us whether the localization results of these two positions are close to the actual distance ( $k$ feet).

\section{Experiments}

In this section we describe three types of experiments we conducted. In all cases, our results are trace-driven. That is, we collected the fingerprints in a real environment, and then performed the localization off-line by running different localization algorithms using the collected fingerprints. When an algorithm required a training phase, e.g., training data for $\mathrm{BN}$ or a signal map for RADAR, we always use the points from the center placements. We use a leave-one-out methodology for computing the accuracy and stability CDFs. That is, if applicable, we give an algorithm a training or signal map with measured or interpolated fingerprints from the center locations and give it a fingerprint from an unknown location to localize. Note that some versions of BN do not use fingerprints with known coordinates; we describe these later in Section IV-D.

Horizontal and Vertical Movements Experiments. The first set of experiments we performed examined accuracy and stability as a function of small-scale movements around a given testing spot. We tested both these metrics in the horizontal plane, i.e., $(x, y)$, using the desk-level fingerprints including the center, north, south, east, west, vertical and parallel placements. In the vertical, i.e., $z$ plane, we used the floor, center and shoulder placements. In both cases, the center location serves as the "original" $p_{1}$, and the other positions are the additional small-scale movements.

Data Averaging and Non-averaging Experiments. An important open question is if landmarks should aggregate the RSS readings from the different antennas or a localization algorithm should use directly the raw RSS from each antenna. In our case, the simple aggregation scheme we examined was to perform an averaging between the antennas at a given landmark; more complex schemes are left as our future work.

We derived a systematic way to evaluate the localization performance under the cases of a single antenna, two antennas, and three antennas by either using the raw RSS readings from each individual antenna or averaging the RSS readings over two or three antennas from a landmark position. Table III summarizes various antenna combinations we consider here. In order to insure the generality of the results, we tried different combinations of 1 and 2 antennas. Each combination is given a specific name as shown in Table III.

Distribution Experiments. A third class of experiments investigated the impact of assuming that RSS data follows a Gaussian distribution. Such assumptions are quite common among localization algorithms. For example, both the ABP and $\mathrm{BN}$ algorithms assume the data follows a Gaussian distribution.

In order to measure the impact of this assumption, we generated a data set of fingerprints we call the Gaussian one. To generate this distribution, we used the signal propagation constants fitted to a simple propagation model, described in Section IV-A. The model defines the mean RSS that should be observed given the distance between the mobile and the landmark. To compute the variance, we used the variance of the fitted distribution. We then generated fingerprints using a Gaussian distribution for each RSS reading in a fingerprint.

\section{RESUlts}

In this section, we describe our results. In order to get some intuition if multiple antennas do help average out small-scale effects, we first describe a small experiment examining the goodness of fit of RSS data to a theoretic model. We then present the accuracy and stability results for RADAR, Area Based Probability (ABP), and Bayesian Networks (BNs). For each algorithm, we show overall accuracy and stability results for small-scale movements, when averaging and not averaging the antenna data, and when applying the Gaussian distribution.

\section{A. Impact on Free Space Models}

In this section, we look for evidence on how multiple antennas "smooth out" the effects of small-scale variations in signal strength. An intuitive definition of "smooth out" is that the change in RSS does not vary much with a change in location. We experimented with several metrics examining rates of change in signal space vs. location when using multiple antennas, however, we did not find them grounded with sufficient theoretical foundations to use them.

Our metric is to examine how well readings from multiple antennas fit a simple propagation model. Recall that in free space, signal power decays approximately linearly with log distance. Specifically, signal strength $S$ can be described by the following propagation model:

$$
S=b_{0}+b_{1} \log (D)
$$

where in equation (1), $b_{0}, b_{1}$ are propagation constants of the model and $D$ is the Euclidean distance between the transmitter and the receiver. We call such a log-linear model a free space model.

Our approach is to add multiple antennas, and then observe the goodness of fit of the data to a best fit free-space model. The goodness of fit is observable as the coefficient of determination or $R^{2}$. Recall that $R^{2}$ can take values from 0 to 1 , with a value of 1 indicating a perfect fit to the model, and a value close to 0 indicating a poor fit. Table IV describes various cases of antenna combinations: a single antenna, averaging on two antennas, and averaging on three antennas.

Figure 2 presents the $R^{2}$ for the five landmark locations. For positions $A, B, C, D$ by averaging the RSS of all three antennas (3-antenna-avg), the RSS data set achieves the best fit, with $R^{2}$ around 0.8 . We also see that averaging gives a better fit when compared to the fit of single antennas. Thus, 


\begin{tabular}{|l|l|}
\hline Antenna Combination & Description \\
\hline \hline 1-antenna-odd & $\begin{array}{l}\text { RSS from antenna with odd ID } \\
\text { (i.e., } 1,3,5,7,9)\end{array}$ \\
\hline 1-antenna-even-small & $\begin{array}{l}\text { RSS from antenna with small, even ID } \\
\text { (i.e., } 2,4,6,8,10)\end{array}$ \\
\hline 1-antenna-even-large & $\begin{array}{l}\text { RSS from antenna with large, even ID } \\
\text { (i.e., 12, 14, 16, 18, 20) }\end{array}$ \\
\hline 2-antenna-avg & $\begin{array}{l}\text { average } \mathrm{RSS} \text { from antennas with even IDs } \\
\text { (i.e., avg }(2,12), \text { avg(4, 14), avg(6, 16), avg(8, } \\
\text { 18), avg(10, 20)) }\end{array}$ \\
\hline 3-antenna-avg & $\begin{array}{l}\text { average } \mathrm{RSS} \text { from all three antennas } \\
\text { (i.e., avg(1, 2, 12), avg(3, 4, 14), avg(5, 6, 16), } \\
\text { avg(7, 8, 18), avg(9, 10, 20)) }\end{array}$ \\
\hline
\end{tabular}

TABLE IV

Variability antenna combinations for a given landmark position.

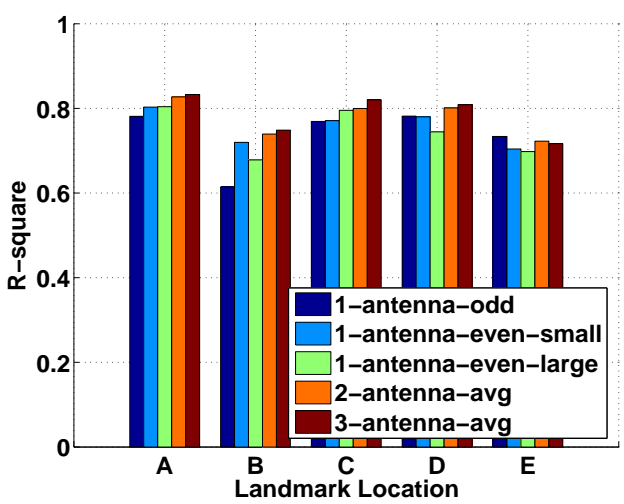

Fig. 2. Goodness of fit of real RSS to the signal propagation model.

adding multiple antennas does improve the data fit to a simple free-space model, although the effect is not very large.

\section{B. RADAR}

The RADAR algorithm is a classic scene-matching localization algorithm [1]. RADAR requires a signal map, which is a set of fingerprints with known $(x, y)$ locations. Given a fingerprint with an unknown location, i.e., one to localize, RADAR returns the $x, y$ of the closest fingerprint in the signal map to the one to localize, where "closest" is defined as the Euclidean distance of the fingerprints to each other in an $N$-dimensional "signal space" with $N$ landmarks [19]. That is, it views the fingerprints as points in an $N$-dimensional space, where each landmark forms a dimension, and returns the corresponding $x, y$ of the closest point. In our experiments that follow, the distance between the points in the signal map is about $5-10 \mathrm{ft}$.

Accuracy. Figure 3 presents the localization error CDFs of RADAR for the antenna combinations displayed in Table III. Figure 3(a) shows the localization error for the center position at the desk level. We see that using 3 antennas at a landmark position results in a better performance than using only 1 antenna or using 2 antennas. Specifically, we found that at the error of $10 \mathrm{ft}$, the probability increases from $42 \%$ for the 1 - antenna case to $70 \%$ under the 3 - anntena - avg case, and at the error of $20 \mathrm{ft}$, the probability increases from $77 \%$ for the 1 - antenna case to $90 \%$ for the 3 - antenna - avg case. The overall improvement for the median error is $20 \%$

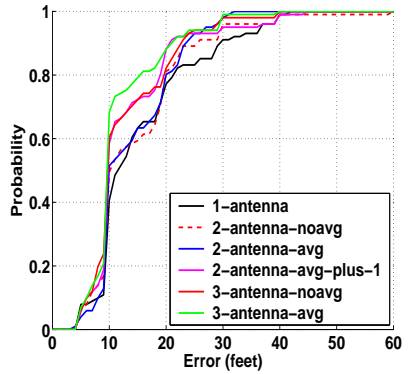

(a) Desk, Center

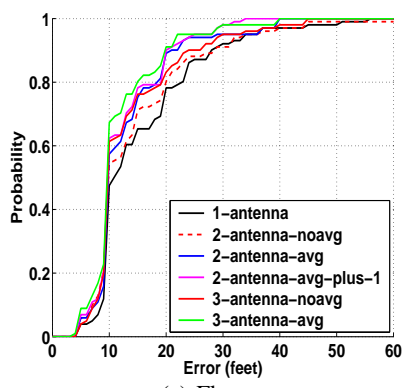

(c) Floor

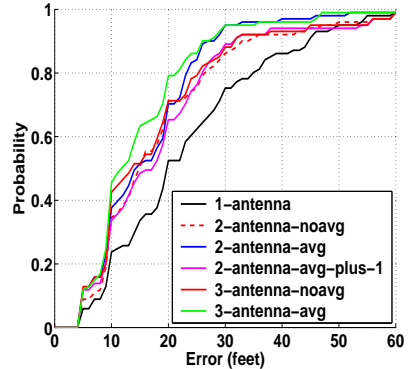

(b) Gaussian

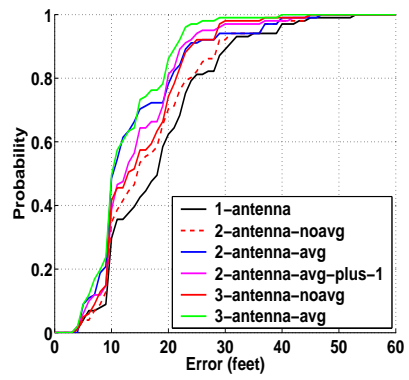

(d) Shoulder
Fig. 3. Localization error CDFs using RADAR.

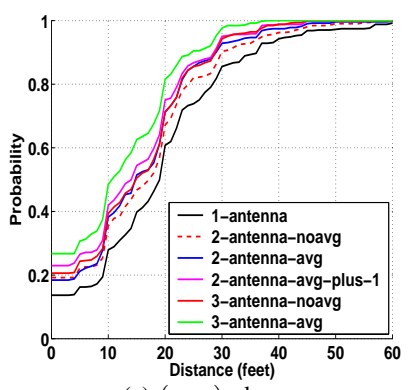

(a) $(x, y)$ plane

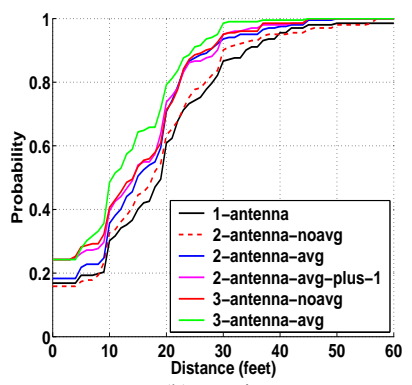

(b) $z$-axis
Fig. 4. Localization stability using RADAR.

and the 90th percentile error is $29 \%$.

Figures 3(c) and 3(d) are the corresponding error CDFs for the floor and shoulder positions respectively. We observe comparable performance behavior to that at the desk level under multiple antennas. Further, the long CDF tails in Figure 3(c) indicate we have larger maximum localization errors at the floor level, compared to the desk and shoulder level. This is due to the fact that at the floor level the signal suffers from shadowing.

We further studied the localization performance for RADAR when modeling the RSS as a Gaussian distribution, which was discussed in Section III-C. The resulting localization errors are presented in Figure 3(b). We see that the error CDFs follow the same trend as in Figure 3(a), but with qualitatively worse performance than the single antenna case. This indicates that when using a fingerprint matching method for localization, modeling the RSS as a Gaussian distribution provides consistent results with real experimental data.

Stability. Figure 4 presents the localization stability for RADAR when using multiple antennas. Examining the distance CDFs at the $(x, y)$ plane as shown in Figure 4(a), we found that the total percentage of the small-scale movements of the mobile device being localized back to the same esti- 
mated position when the mobile device resides at its original center position increases from $13.7 \%$ for a single antenna to $26.7 \%$ when averaging the RSS from 3 antennas at one landmark position. A $100 \%$ improvement is achieved in this case. Further, the stability at the 50th percentile moves from $19 \mathrm{ft}(1-$ antenna $)$ to $11 \mathrm{ft}(3$ - antenna - avg), indicating a $42 \%$ improvement, whereas the 90th percentile achieves a comparable 30\% improvement.

Moreover, as shown in Figure 4(b) the stability of the mobile device movement along the $z$-axis exhibits similar behavior. This is very encouraging as better localization stability strongly indicates that using multiple low-cost antennas for improving localization performance is effective.

\section{Area Based Probability}

Area Based Probability (ABP) utilizes an Interpolated Map Grid (IMG) to interpolate the signal map to cover the entire experimental floor. The floor is divided into a regular grid of equal-sized tiles. Because direct measurement of the fingerprint for each tile is expensive and prohibitive for fine-grained tiles, we use an interpolation approach. The goal of using an IMG fitting is to derive an expected RSS fingerprint for each tile from the data set that would be similar to an observed one.

ABP returns a set of tiles bounded by a probability that the mobile device is within the returned tile set. The probability is called the confidence $\alpha$ and it is adjustable by the user. We used a tile size of 10in $\times 5$ in, which is comparable to the distance between the antennas at a landmark location ( 1 or 2 $\mathrm{ft}$ ). ABP assumes the distribution of RSS for each landmark follows a Gaussian distribution with mean as the expected value of RSS reading vector $\mathbf{s}$. The Gaussian random variable from each landmark is independent. ABP then computes the probability of the mobile device being at each tile $L_{i}$, with $i=1 \ldots L$, on the floor using Bayes' rule:

$$
P\left(L_{i} \mid \mathbf{s}\right)=\frac{P\left(\mathbf{s} \mid L_{i}\right) \times P\left(L_{i}\right)}{P(\mathbf{s})}
$$

Given that the mobile device must be at exactly one tile satisfying $\sum_{i=1}^{L} P\left(L_{i} \mid \mathbf{s}\right)=1$, ABP normalizes the probability and returns the most likely tiles/grids up to its confidence $\alpha$ [2]. In order to normalize for accuracy and stability results, we select the tile with the median localization error from the tile set. In all results we show next, the value of the confidence level is $\alpha=0.75$.

Accuracy. Figure 5 shows the localization error CDFs of ABP when using multiple antennas. The 3 -antenna-noavg case tops out the performance. Comparing to the 1 -antenna case, we observe that the median error moves from $7 \mathrm{ft}$ to 2 $\mathrm{ft}$ and the 90th percentile error moves from $16 \mathrm{ft}$ to $4 \mathrm{ft}$ under the 3 - antenna - noavg case. Thus, the location accuracy has an improvement over $70 \%$ for both the median as well as the 90th percentile error when using 3 antennas at a given location.

Examining the $z$-axis, the error CDFs for the floor and shoulder level in Figures 5(c) and 5(d) have qualitatively similar performance to the desk level shown in 5(a). Again, by

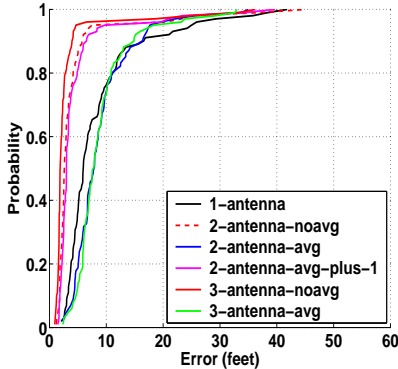

(a) Desk, Center

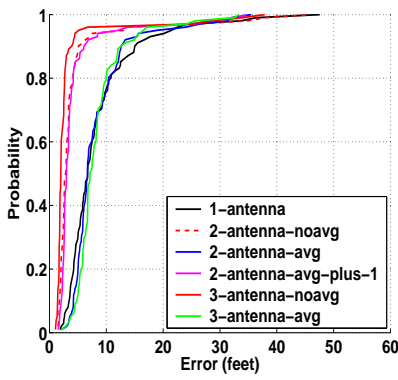

(c) Floor

Fig. 5. Localization error $\mathrm{CDF}$ s using $\mathrm{ABP}$

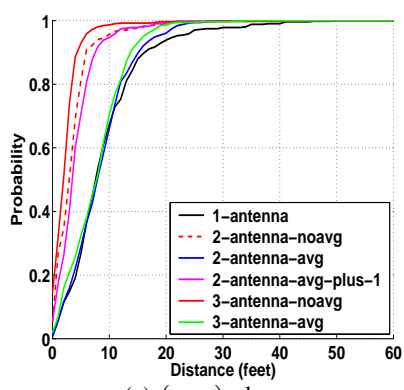

(a) $(x, y)$ plane

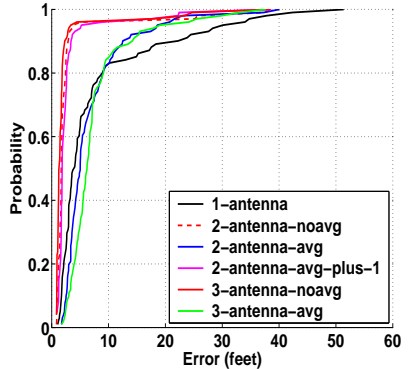

(b) Gaussian

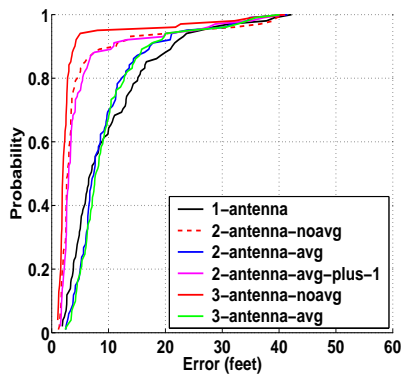

(d) Shoulder
Fig. 6. Localization stability using ABP.

using the RSS readings from each antenna at a given landmark location achieves the best performance. Moreover, we noticed that the CDFs at the floor level only have slightly longer tails than those at the desk and shoulder level. This indicates that by interpolating the signal map across the experimental floor helps smooth out the signal variability and thus reduces the maximum localization errors.

Finally, Figure 5(b) presents the localization errors using the Gaussian data set. We observe consistent better performance under the cases of multiple antennas. The performance of the 3 - antenna - noavg case using the Gaussian simulated data is even better than using the real experimental data.

The above results in location accuracy show that when using the approach of an interpolated signal map with grid size smaller than the distance between 2 adjacent antennas at a landmark location, each antenna is treated as a separate landmark, the 3 -antenna-noavg case has the best performance and improves the localization accuracy over $70 \%$. Hence, by using signal map interpolation, we achieved better location accuracy improvement than using the raw fingerprints in the signal map directly (as RADAR does).

Stability. Figure 6 shows that using multiple antennas, including the cases of 2 -antenna-noavg, 2 - antenna- 


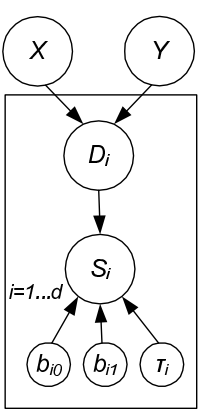

(a) Network $M_{1}$

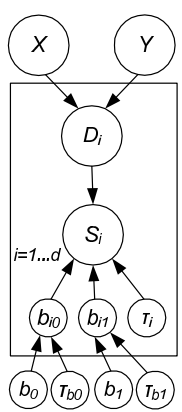

(b) Network $M_{2}$

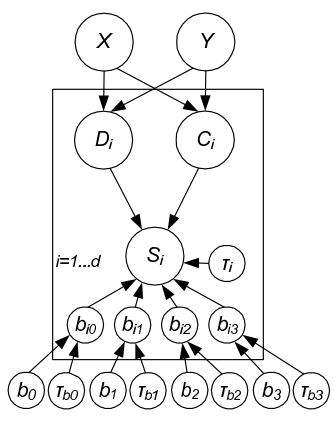

(c) Network $M_{3}$
Fig. 7. Bayesian graphical networks using plate notation.

avg - plus - 1 and 3 - antenna - noavg at a given location helps improve localization stability, with the 3 -antennanoavg case providing the most stability improvement. Notably, the total percentage of testing points, under small-scale movements, localized back to the original localization result (i.e., location estimation at the center) increases from less than $5 \%$ for a single antenna to over $14 \%$ for the case of 3 - antenna - noavg. Similar to RADAR, this is an over $100 \%$ stability improvement. Further, examining Figure 6(a) the stability distance at the 50th percentile moves from $8 \mathrm{ft}$ for the case of 1 -antenna to $2 \mathrm{ft}$ for the 3 -antenna-noavg case, resulting in a stability improvement of $73 \%$. We also observe over $70 \%$ improvement for the 90 th percentile. In the $z$-axis as shown in Figure 6(b), employing multiple antennas at a given location again provides consistent improvement in localization stability.

One effect we observed is that when using signal map interpolation, the cases of averaging the RSS readings from multiple antennas at a given location such as 2-antenna-avg and 3 - antenna - avg have the same localization performance as the single antenna case. This is because for tiny grids (10in $\times$ 5in), averaging RSS at a given location is just like placing a single landmark at a location, which is the same as having a single antenna in a landmark.

\section{Bayesian Networks}

Bayesian Networks (BNs) are graphical models that encode dependencies and relationships among a set of random variables. The vertices of the graph correspond to variables and the edges represent dependencies [20]. As described in [3], the BNs we use encode the relationship between the RSS and a location based on the signal-versus-distance propagation model shown in Equation 1.

Figure 7 presents a series of Bayesian networks called $M_{1}$, $M_{2}$, and $M_{3}$. Each rectangle is a "plate" [3], and shows a part of the network that is replicated. The vertices $X$ and $Y$ represent location, the vertex $S_{i}$ is the RSS reading from the $i$ th landmark, and the vertex $D_{i}$ represents the Euclidean distance between the location specified by $X$ and $Y$ and the $i$ th landmark. The value of $S_{i}$ follows a signal propagation model $S_{i}=b_{0 i}+b_{1 i} \log D_{i}$, where $b_{0 i}, b_{1 i}$ are parameters specific to the $i$ th landmark. The distance $D_{i}=\sqrt{\left(X-x_{i}\right)^{2}+\left(Y-y_{i}\right)^{2}}$ depends on the location

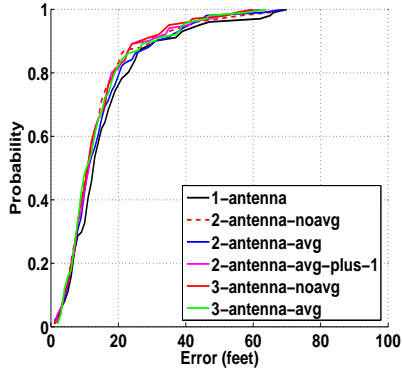

(a) Center: Training $=100$, Testing $=1$

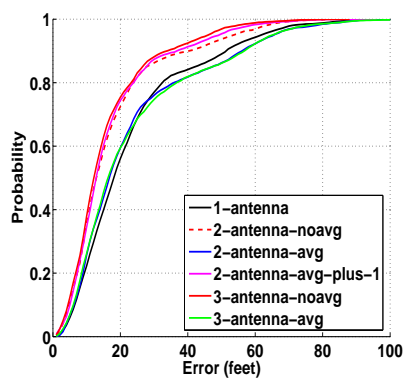

(c) Floor: No training,

$$
\text { Testing }=51
$$

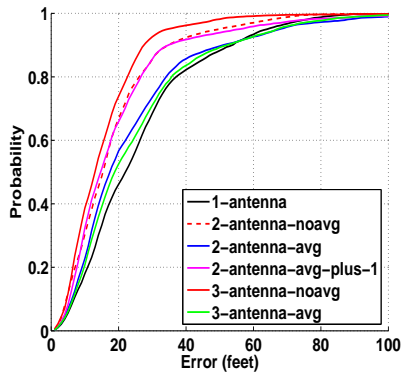

(b) Center: No training, Testing $=51$

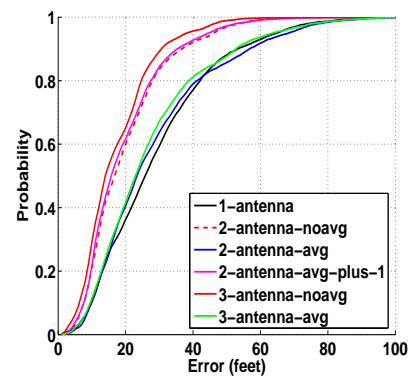

(d) Shoulder: No training,

$$
\text { Testing }=51
$$

Fig. 8. Localization error CDFs using Bayesian network $M_{2}$.

$(X, Y)$ where the signal is transmitted and the coordinates $\left(x_{i}, y_{i}\right)$ of the $i$ th landmark. The networks capture noise and outliers by modeling the $S_{i}$ as a Gaussian distribution around the above propagation model with variance $\tau_{i}$ :

$$
S_{i} \sim N\left(b_{0 i}+b_{1 i} \log D_{i}, \tau_{i}\right)
$$

The parameters $b_{0 i}, b_{1 i}, \tau_{i}$ of the model are unknown, and the training fingerprints as well as the fingerprint vector of the mobile object are used to adjust their value according to the relationships encoded in the network. Through Markov Chain Monte Carlo (MCMC) simulation, BNs compute a probability distribution for the location variables $X$ and $Y$.

Network $M_{1}$ is the simplest amongst the three depicted in Figure 7, and requires a training set in order to give good localization results. Network $M_{2}$ is hierarchical by making the coefficients of the signal propagation model have common parents. As was shown in [3], $M_{2}$ can localize with no training fingerprints, leading to a zero-profiling technique for location estimation. The impact of multiple antennas on zero-profiling is a key effect we tested for, because typically this approach has the measurably worst performance, but has the benefit of not having to collect fingerprints at known locations. Finally, network $M_{3}$ extends $M_{2}$ by incorporating the corridor effect. That is, when a location $(X, Y)$ shares a corridor with a landmark, then the signal strength tends to be stronger along the entire corridor. In this paper, we define "sharing a corridor" as having an $X$ - or $Y$-coordinate within three feet of the corresponding landmark coordinate.

Accuracy. Figure 8 presents the localization error CDFs of $M_{2}$ under multiple antennas. Figure 8(a) shows the error CDFs, when localizing one device (testing=1) in its center position at desk level. We see that all the curves have similar performance, although case 3 - antenna - noavg performs 


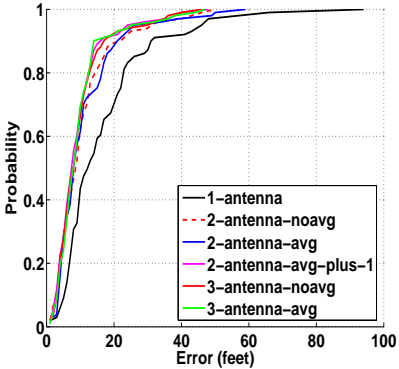

(a) Center: Training $=100$,

Testing $=1$

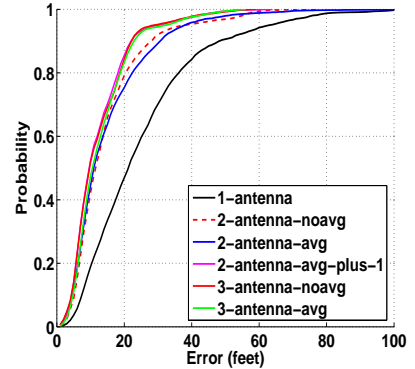

(b) Center: No training,

Testing $=51$
Fig. 9. Gaussian approach: Localization error CDFs using Bayesian network $\mathrm{M}_{2}$.

slightly better than the rest, and 1 - antenna is the worst. The other two networks $\left(M_{1}, M_{3}\right)$ have performance similar to $M_{2}$ under multiple antennas, and their results are omitted here due to space constraints.

Figures 8(b), 8(c), and 8(d) present the error CDFs of $M_{2}$ when localizing 51 devices simultaneously with no training set at the levels of desk, floor, and shoulder respectively. We see that the 3-antenna-noavg case has the best performance for all three positions. The accuracy improvement on the median error between 1 -antenna and 3 -antenna-noavg is $40 \%$ (from $22 \mathrm{ft}$ to $13 \mathrm{ft}$ ) at the desk level, $33 \%$ (from $18 \mathrm{ft}$ to $12 \mathrm{ft}$ ) at the floor level, and $46 \%$ (from $26 \mathrm{ft}$ to $14 \mathrm{ft}$ ) at the shoulder level. Similarly, the same amount of improvement is achieved at the 90th percentile of the error CDFs. Conclusively, our empirical results suggest that more antennas per landmark location primarily help improve the localization accuracy of Bayesian networks when there is no training set.

Another important observation is that 3 antennas per landmark location, case 3 -antenna-noavg, makes the localization error when locating multiple mobile devices comparable to that when locating a single device. Specifically, from Figures 8(a) and 8(b) we see that the median and the 90th percentile errors for testing $=1$ and testing $=51$ are of the same magnitude for 3 - antenna-noavg.

Figure 9 depicts the localization accuracy of Bayesian Network $M_{2}$ when using the Gaussian simulated data set. We observe that in both figures the different antenna combinations can be placed into three groups based on their performance: (a) 3 - antenna - noavg, 3-antenna-avg, 2 - antenna - avg - plus - 1, (b) 2 - antenna - noavg, 2 -antenn-avg, and (c) 1-antenna. The first group has the best performance, whereas the last the worst. Intuitively, this kind of grouping should be expected, since BNs assume that the RSS follows a Gaussian distribution, and thus the averaged RSS of antennas that belong to the same landmark position is also Gaussian [21]. Therefore, unlike with real experimental data, BNs perform similarly either we average or not average the RSS of the multiple antennas at a given landmark location.

Stability. Figure 10 presents localization stability CDFs for Bayesian network $M_{2}$. The stability results for the other networks $\left(M_{1}, M_{3}\right)$ are similar and hence omitted. Figure 10(a) shows that when localizing one mobile device, the more antennas per landmark location, the better the stability. The stability improves by $36 \%$ at the 50th percentile, moving from $11 \mathrm{ft}(1-$ antenna $)$ to $7 \mathrm{ft}(3-$ antenna - noavg $)$, and $33 \%$ at the 90th percentile, moving from $21 \mathrm{ft}(1-$ antenna) to 14 $\mathrm{ft}(3-$ antenna - noavg $)$. However, the difference between the cases of 3 - antenna - noavg and 3 - antenna - avg is negligible. In general, we noticed that when our networks use training data, averaging or not averaging the RSS from multiple antennas at a given location does not make any difference on the localization stability, regardless of the number of multiple devices we localize simultaneously.

Figures 10(b) and 10(c) show that when no training data is used, case 3 -antenna - noavg has the best performance. Moreover, the more antennas the better, but 3 - antenna noavg gives better stability than 3 -antenna - avg. At the desk level, the median of stability improves by $43 \%$, from 16 $\mathrm{ft}(1-$ antenna $)$ to $9 \mathrm{ft}(3-$ antenna - noavg $)$, and the 90 th percentile by $44 \%$, from $36 \mathrm{ft}(1-$ antenna) to $20 \mathrm{ft}(3-$ antenna-noavg). Along the $z$-axis, there is an improvement of $40 \%$ at the median and $34 \%$ at the 90th percentile.

\section{DiscusSiON}

One observation is that the distance between multiple antennas at a landmark location has important effects on localization algorithms. The signal map in RADAR uses the measured fingerprints directly. In Figure 3 the location accuracy in the 3 - antenna - avg case is slightly better than the 3 - antenna - noavg case. We believe this is because in our study the distance between two antennas is small, only 1-2 ft away from each other, while the testing points are about 5-10 ft away from each other, which is a magnitude of 5 times larger. The fine-grained RSS differences at 3 antennas will not affect the coarse-grained fingerprint matching. As a result, the 3 separate antennas at one landmark location will not be treated as 3 separate landmarks by the algorithm when performing fingerprint matching in the signal space. Additionally, averaging the RSS from 3 antennas reduces the RSS variability and thus provides the best performance when working with algorithms dealing with fingerprints directly.

On the other hand, ABP uses an interpolated signal map and a tile size of 10 in $\times 5$ in, which is comparable to the distance between two antennas at a landmark location. We found that using the RSS from each individual antenna (3 - antennanoavg), which treats each antenna as a separate landmark, achieves the best improvement in both location accuracy and stability as shown in Figures 5 and 6.

In general, we believe that the ratio of the granularity of the distances between the antennas at a landmark and the distances used to collect training/testing fingerprints can have a significant impact on the results of either averaging or not averaging RSS of multiple antennas. A full characterization of this effect is left as our future work.

\section{CONCLUSION}

By employing multiple receivers spaced closely at a given location, we investigated the impact on wireless localization under various cases of multiple antennas. We performed a 


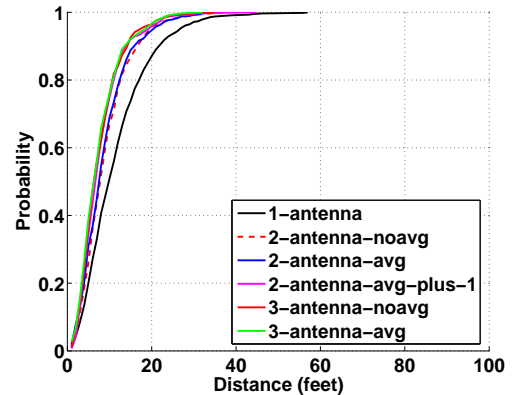

(a) $(x, y)$ plane: Training $=100$, Testing $=1$

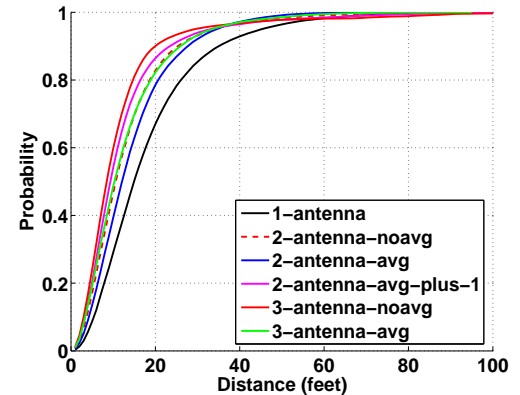

(b) $(x, y)$ plane: No training, Testing $=51$

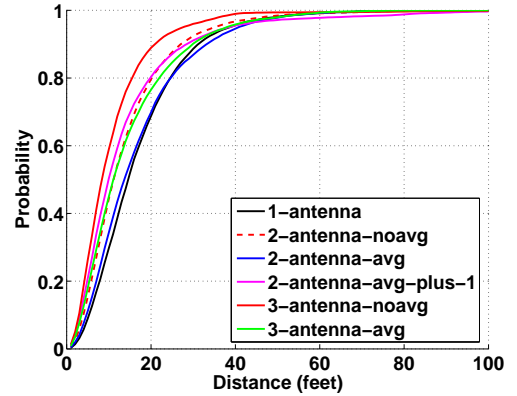

(c) $z$-axis: No training, Testing $=51$

Fig. 10. Localization stability using Bayesian network $M_{2}$.

trace-driven study on an 802.11 wireless testbed in a real office environment. Through a signal variability study, we found that adding additional antennas helps average out small-scale environmental effects.

We then studied the performance of a representative set of localization algorithms, in localization accuracy and stability, when using multiple antennas. We found that all algorithms under study improved their absolute accuracy by either averaging or not averaging the RSS from multiple antennas at a given location. Specifically, both the median error as well as the 90th percentile error can be reduced up to $70 \%$. One important observation is that the ratio of the distance between the training/testing points to the distance between multiple antennas at a landmark location affects the localization accuracy. Notably, localization schemes utilizing the tiny grids obtained by the signal map interpolation achieved the best performance improvement when treating each individual antenna at a given location as distinct landmarks.

Finally, our investigation of the localization stability when there are small-scale movements of a mobile device shows that multiple antennas help improve localization stability significantly, up to $100 \%$ improvement over the regular single antenna case. In summary, we found that adding multiple antennas gives substantial performance benefits for localization that are worth the costs.

\section{ACKNOWLEDGMENTS}

We would like to acknowledge support from NSF grants CNS-0448062 and CNS-0335244.

\section{REFERENCES}

[1] P. Bahl and V. N. Padmanabhan, "RADAR: An In-Building RF-Based User Location and Tracking System," in Proceedings of the 19th IEEE International Conference on Computer Communications (INFOCOM), March 2000.

[2] E. Elnahrawy, X. Li, and R. P. Martin, "The Limits of Localization Using Signal Strength: A Comparative Study," in Proceedings of the First IEEE International Conference on Sensor and Ad hoc Communcations and Networks (SECON), October 2004.

[3] D. Madigan, E. Elnahrawy, R. Martin, W. Ju, P. Krishnan, and A. Krishnakumar, "Bayesian Indoor Positioning Systems," in Proceedings of the 24th IEEE International Conference on Computer Communications (INFOCOM), March 2005, pp. 324-331.

[4] N. Priyantha, A. Chakraborty, and H. Balakrishnan, "The Cricket Location-Support System," in Proceedings of the 6th ACM International Conference on Mobile Computing and Networking (MOBICOM), Boston, MA, Aug. 2000.
[5] K. Lorincz and M. Welsh, "MoteTrack: A Robust, Decentralized Location Tracking System for Disaster Response," 2006, to Appear in Springer Personal and Ubiquitous Computing, Special Issue on Location and Context-Awareness.

[6] Y. Chen, J. Francisco, W. Trappe, and R. P. Martin, "A Practical Approach to Landmark Deployment for Indoor Localization," in Proceedings of the Third Annual IEEE Communications Society Conference on Sensor, Mesh and Ad Hoc Communications and Networks (SECON), September 2006.

[7] H. Lim, L. Kung, J. Hou, and H. Luo, "Zero-Configuration, Robust Indoor Localization: Theory and Experimentation," in Proceedings of the IEEE International Conference on Computer Communications (INFOCOM), March 2006.

[8] D. Lymberopoulos, Q. Lindsey, and A. Savvides, "An Empirical Analysis of Radio Signal Strength Variability in IEEE 802.15.4 Networks using Monopole Antennas," Yale Univeristy, ENALAB, Technical Report 050501, 2006.

[9] H. Hashemi, "The Indoor Radio Propagation Channel," in Proceedings of the IEEE, vol. 81, no. 7, July 1993.

[10] L. C. Godara, "Applications of Antenna Arrays to Mobile Communications, Part I: Performance Improvement, Feasibility, and System Considerations," in Proceedings of the IEEE, vol. 85, no. 7, July 1997.

[11] J. H. Winters, J. Salz, and R. D. Gitlin, "The Impact of Antenna Diversity on the Capacity of Wireless Communication Systems," IEEE Transactions on Communications, vol. 42, no. 234, February 1994.

[12] V. A. N. Barroso, M. J. Rendas, and J. P. Gomes, "Impact of Array Processing Techniques on the Design of Mobile Communications Systems," in Proceedings of the IEEE 7th Mediterranean Electrotechnical Conference, Antalya, Turkey, April 1994.

[13] M. Mizuno and T. Ohgane, "Application of Adaptive Array Antennas to Radio Communications," Electronics and Communications in Japan (Part I: Communications), vol. 77, no. 2, 1994.

[14] M. Barrett and R. Arnott, "Adaptive Antennas for Mobile Communications," Electronics and Communication Engineering Journal, vol. 6, August 1994.

[15] S. C. Swales, M. A. Beach, D. J. Edwards, and J. P. McGeehan, "The Performance Enhancement of Multibeam Adaptive Base-Station Antennas for Cellular Land Mobile Radio Systems," IEEE Transactions on Vehicular Technology, vol. 39, no. 1, February 1990.

[16] M. Goldburg and R. H. Roy, "The Impacts of SDMA on PCS System Design," in Proceedings of the IEEE 3rd Annual International Conference on Universal Personal Communications, San Diego, CA, 1994.

[17] M. Chryssomallis, "Smart Antennas," IEEE Antennas and Propagation Magazine, vol. 42, no. 3, June 2000.

[18] Y. Chen, E. Elnahraway, J.-A. Francisco, K. Kleisouris, X. Li, H. Xue, and R. P. Martin, "Grail: General Real Time Adaptable Indoor Localization," in Proceedings of the 4th ACM Conference on Embedded Networked Sensor Systems (SENSYS), Demo Abstract, Nov. 2006.

[19] Y. Chen, K. Kleisouris, X. Li, W. Trappe, and R. P. Martin, "The Robustness of Localization Algorithms to Signal Strength Attacks: A Comparative Study," in Proceedings of the International Conference on Distributed Computing in Sensor Systems (DCOSS), June 2006, pp. 546563.

[20] A. Gelman, J. B. Carlin, H. S. Stern, and D. B. Rubin, Bayesian Data Analysis, 2nd ed. Chapman and Hall, 2004.

[21] G. Casella and R. L. Berger, Statistical Inference. Belmont, California: Duxbury Press, 1990. 